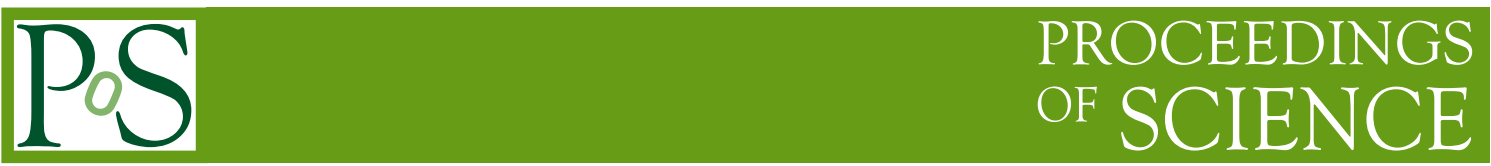

\title{
Calorimetry at Very Forward Rapidity
}

\author{
Edward Scott* on behalf of the CMS Collaboration \\ Imperial College London, $U K$ \\ E-mail: ed.scottacern.ch
}

The CMS experiment at CERN will undergo significant improvements during the so-called PhaseII Upgrade to cope with a 10-fold increase in integrated luminosity with the High Luminosity LHC (HL-LHC) era. A particularly challenging environment is the forward region, where the combination of extremely high radiation levels (fluence and ionising dose) coupled with up to 200 simultaneous pileup events necessitates new technologies and methodologies. The CMS collaboration is designing a High Granularity Calorimeter (HGCAL) to replace the existing endcap calorimeters. It is a sampling calorimeter, featuring unprecedented transverse and longitudinal readout segmentation for both electromagnetic (CE-E) and hadronic (CE-H) compartments. This will facilitate particle-flow calorimetry, where the fine structure of showers can be measured and used to enhance pileup rejection and particle identification, whilst still achieving good energy resolution. The CE-E and a large fraction of CE-H will use silicon as active detector material. The sensors will be of hexagonal shape, maximising the available 8-inch circular wafer area. The lower-radiation environment will be instrumented with scintillator tiles with on-tile SiPM readout. In addition to the hardware aspects, the reconstruction of signals - both online for triggering and offline - is a quantum leap from existing detectors. We present the current status of the HGCAL, including its design and expected performance and the challenges ahead.

Sixth Annual Conference on Large Hadron Collider Physics (LHCP2018)

4-9 June 2018

Bologna, Italy

${ }^{*}$ Speaker. 


\section{Introduction}

The LHC at CERN is currently in its final year of Run 2, producing collisions at a centre-of-mass energy $(\sqrt{s})$ of $13 \mathrm{TeV}$ at an instantaneous luminosity of up to $1.7 \times 10^{34} \mathrm{~cm}^{-2} \mathrm{~s}^{-1}$. In order to maximise its physics reach, a comprehensive upgrade of the LHC to become the High-Luminosity LHC (HL-LHC) is planned to take place during the third long shutdown period, between 2024 and 2026. The instantaneous luminosity at the HL-LHC will increase to a nominal $5 \times 10^{34} \mathrm{~cm}^{-2} \mathrm{~s}^{-1}$, corresponding to a planned integrated luminosity of $3000 \mathrm{fb}^{-1}$ at $\sqrt{s}=14 \mathrm{TeV}$ collected by the mid-2030s. With an average of 140-200 pileup (PU) events per bunch crossing, and a greatly increased radiation dose and fluence, the HL-LHC environment will be significantly harsher than the LHC at present. This necessitates an extensive upgrade of the CMS detector [1] to avoid extensive radiation damage and maintain the excellent level of performance exhibited in Run 2. One part of this upgrade will be the replacement of the current CMS endcap calorimeters with the high granularity calorimeter (HGCAL) [2].

\section{The High Granularity Calorimeter}

\subsection{Key requirements of the HGCAL}

Simulations indicate that the HGCAL will be required to withstand up to $2 \mathrm{MGy}$ of total radiation dose, together with a maximum fluence of around $10^{16} \mathrm{neq} / \mathrm{cm}^{2}$. Studies performed in recent years have shown that silicon sensors and the associated electronics retain acceptable performance after exposure to this level of radiation, and have hence been chosen as the most reliable active material for the majority of the calorimeter. The remaining parts of the detector in lower-radiation regions will instead use cheaper plastic scintillator tiles with silicon photomultipliers (SiPMs).

To maintain performance throughout the operation of the HL-LHC it is necessary to inter-calibrate cells to the level of a few percent. This can be achieved with a sufficiently high signal-to-noise ratio ( $\mathrm{S} / \mathrm{N}$ ) for minimum-ionising particles (MIPs), after $3000 \mathrm{fb}^{-1}$. Consequently small silicon cells with low capacitance are required, resulting in high lateral granularity. Fine lateral granularity also has many benefits for physics performance, including the ability to separate nearby showers, identify narrow jets such as those originating from vector boson fusion (VBF), and minimise the amount of pileup entering energy measurements. To take advantage of this fine segmentation the calorimeter is required to be dense, thereby preserving the compactness of showers in the transverse direction. Similarly, fine longitudinal granularity facilitates precise energy measurements, as well as enabling discrimination between different types of shower using the depth profile. These features are particularly important within the CMS particle flow reconstruction paradigm [3]. A detector producing three-dimensional images of showers would provide powerful separation of electromagnetic, charged hadronic and neutral hadronic components, facilitating more precise energy measurements, particularly of jets.

In addition, the HGCAL should be able to perform precise timing measurements enabling excellent pileup rejection, and provide an input to the level one trigger decision. The proposed design specification that captures all of these desired features is described in the following section. 


\subsection{Design parameters}

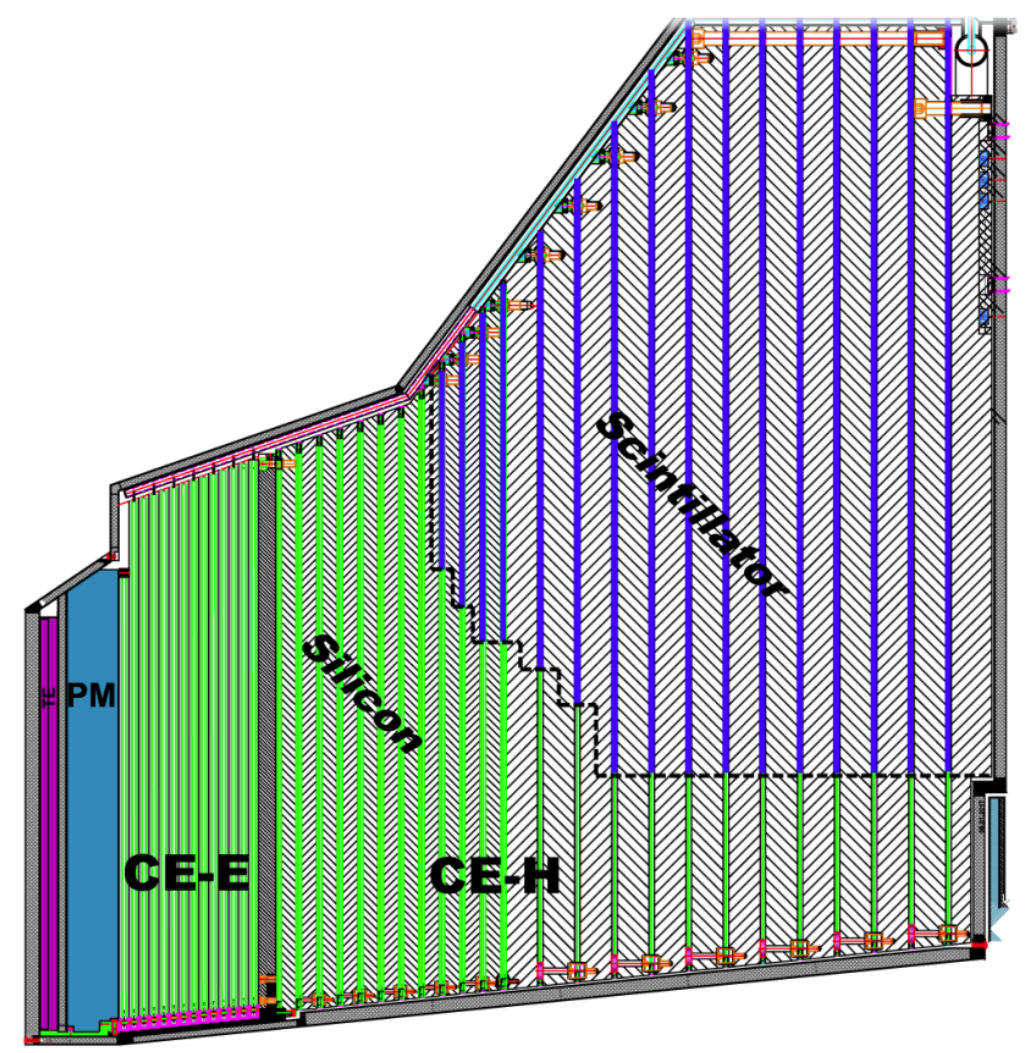

Figure 1: A schematic of the HGCAL. Adapted from [2].

An overview of the HGCAL design is presented in Figure 1. The HGCAL is composed of an electromagnetic and a hadronic section, called the CE-E and CE-H respectively, covering the pseudorapidity range $1.5<|\eta|<3.0$. The CE-E comprises 28 layers with hexagonal silicon sensors as the active element. The total depth, including the neutron moderator layer at the front, is $34 \mathrm{~cm}$, which corresponds to approximately $26 X_{0}$ and $1.7 \lambda$. Absorbers are made of $\mathrm{WCu}$ and $\mathrm{Cu}$ plates are used for cooling. All layers of the CE-E are used for energy measurements, but alternate layers give inputs to the $\mathrm{L} 1$ trigger primitive formation.

The CE-H is formed of 12 layers with 35mm thick stainless steel (SS) absorber and another 12 with $68 \mathrm{~mm}$ thick SS absorber, contributing an additional $9 \lambda$ in depth. The active medium in the $\mathrm{CE}-\mathrm{H}$ varies as a function of depth and radius, and is determined by the radiation level. In regions of sufficiently low fluence (those which are furthest back and furthest from the beam-pipe), plastic scintillator tiles are used with SiPM readout. Further detail on the design specifications of the HGCAL can be found in [2].

\section{Object reconstruction}

The HGCAL's intrinsic performance measuring the energy of electromagnetic showers is modelled 

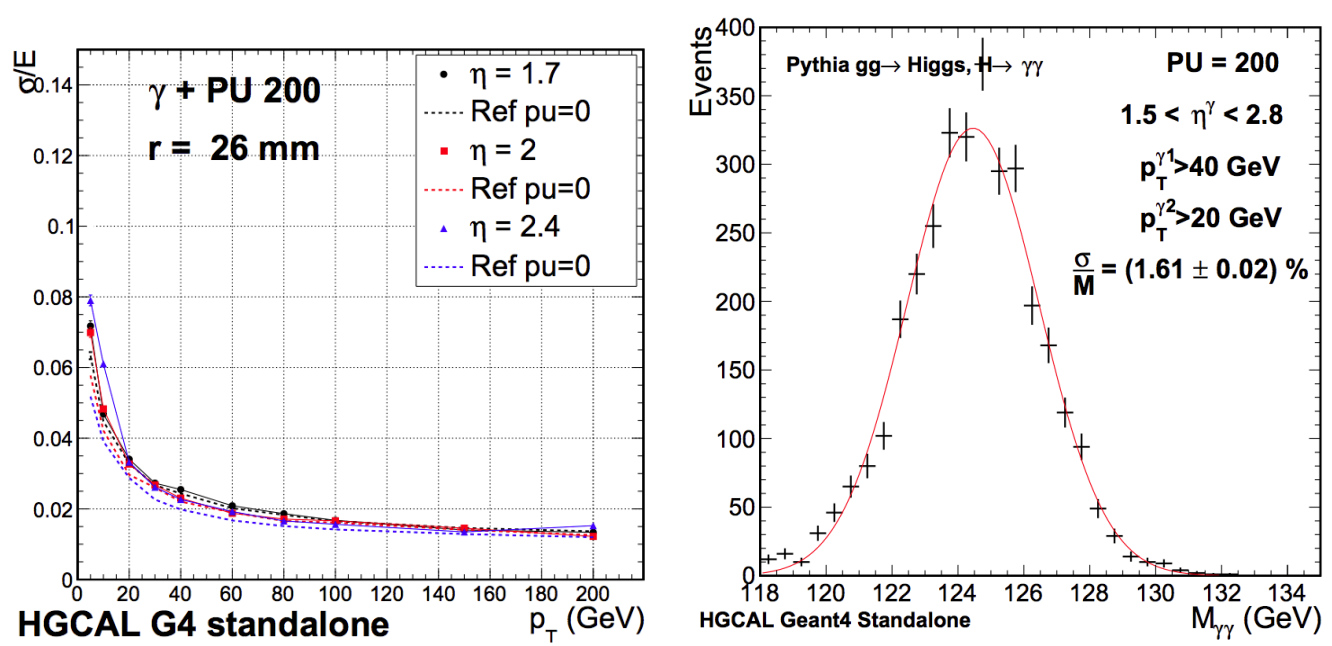

Figure 2: Left: the intrinsic energy resolution for single photons in PU200. The energy is estimated by summing all deposits within a $26 \mathrm{~mm}$ radius of the generated particle axis. Right: the intrinsic diphoton mass resolution of the HGCAL in simulated $H \rightarrow \gamma \gamma$ events where both photons are within the fiducial region of the HGCAL. Both taken from [2].

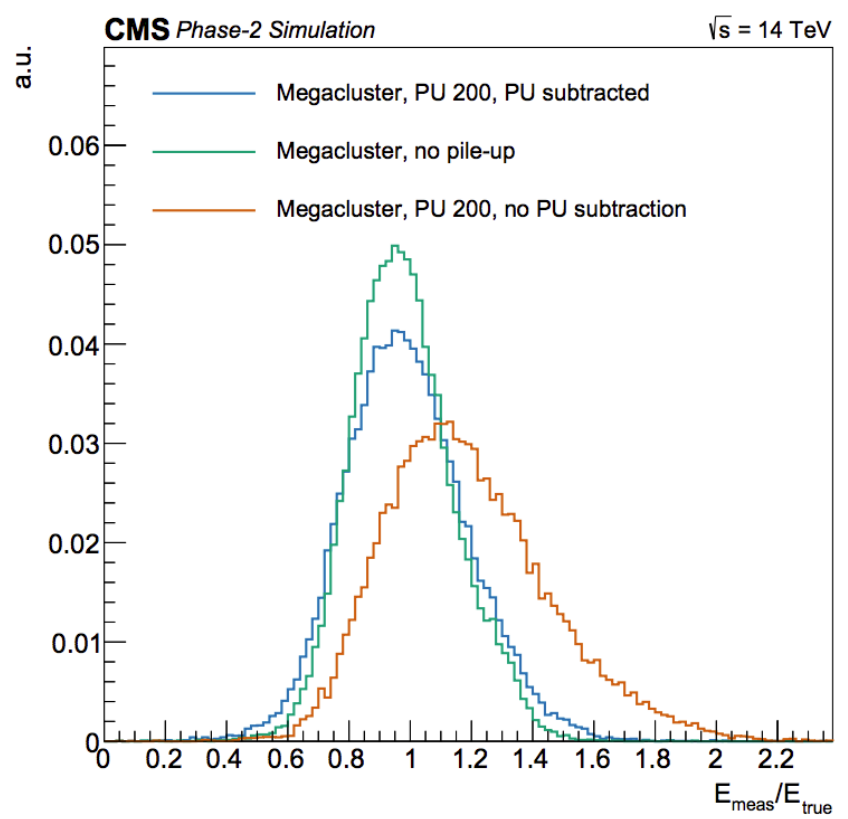

Figure 3: The energy resolution for single $p_{T}=25 \mathrm{GeV}$ pions reconstructed using the megaclustering algorithm, before and after pileup subtraction. Taken from [2].

using a dedicated Geant4 simulation with pileup corresponding to an average of 200 interactions per bunch crossing. Energy deposits in a radius of $26 \mathrm{~mm}$ around a single unconverted photon are summed to estimate the energy resolution, which is shown in Figure 2. The left plot shows that the resolution is approximately constant as a function of $\eta$, and robust against pileup, with only a very small degradation in resolution between PU0 and PU200. This intrinsic performance is 
further demonstrated by using this method to reconstruct unconverted photon pairs from simulated $H \rightarrow \gamma \gamma$ decays, where both photons are contained within the fiducial region of the HGCAL and the vertex location is assumed to be known exactly. The resulting diphoton mass distribution is shown in Figure 2, with resolution of around $1.8 \mathrm{GeV}$. This value is comparable to the expected resolution of the upgraded CMS barrel calorimeter, representing a substantial improvement relative to Run 2.

The HGCAL provides more detailed shower information than existing CMS detectors, and it is envisaged that eventually a sophisticated four-dimensional particle flow approach will be used to incorporate as much of this information as possible. In the meantime, more straightforward approaches to reconstruction have been developed in order to understand which approaches are feasible and to produce object and physics-level results that demonstrate the potential of the detector.

Furthermore, no use is yet made of the intrinsic timing capabilities of the silicon sensors, which will be invaluable in reducing the amount of out-of-time pileup at the HL-LHC. The current method begins by clustering hits in each two-dimensional (2D) layer independently, using an imaging algorithm [4]. These 2D or layer clusters are then associated together in depth to form so-called multiclusters. This step is performed by starting with the highest energy $2 \mathrm{D}$ cluster, then adding any other clusters sufficiently close in $\eta-\phi$ space. It is anticipated that this two-step process could be improved by performing 3D clustering directly, but this remains to be studied in detail.

Electromagnetic objects are then formed using a superclustering procedure very similar to the one utilised in Run 2, collecting together showers which have been spread out in the $\phi$ by the magnetic field. Electrons defined in this way are used to test the ability of the HGCAL to discriminate between signal and background processes. Lateral and longitudinal shower shape variables, along with tracking information, are used as inputs to a Boosted Decision Tree (BDT) classifier. For a 95\% signal efficiency, the background efficiency is $1 \%$ for electrons with $p_{T}>20 \mathrm{GeV}$, comparable to the Run 2 value. An improvement in performance is seen when both lateral and longitudinal shape variables are added to a classifier using track-only information. Additional studies applying the anti- $k_{T}$ algorithm directly on calorimeter hits to form jets have also been performed. Constructing a pileup jet identification using just two variables, one each related to the lateral and longitudinal jet shape, was shown to be permit an acceptable L1 trigger rate of $10 \mathrm{kHz}$ for a signal efficiency of $80 \%$.

Single hadrons were reconstructed using a so-called megaclustering procedure, where multiclusters within a truncated cone are combined to form the object. For these more dispersed hadronic showers, the resolution substantially improves once the contribution of pileup is subtracted. The PU subtraction was implemented by removing the total energy of a similar cone randomly rotated in $\phi$. The energy resolution for a single pion with $p_{T}=25 \mathrm{GeV}$ before and after the subtraction is shown in Figure 3. The megaclustering algorithm is shown to yield adequate energy resolution of around 20\%. It is also robust against pileup, showing a modest worsening between PU0 and PU200 that decreases quickly as a function of $p_{T}$. 


\section{Expected physics performance}

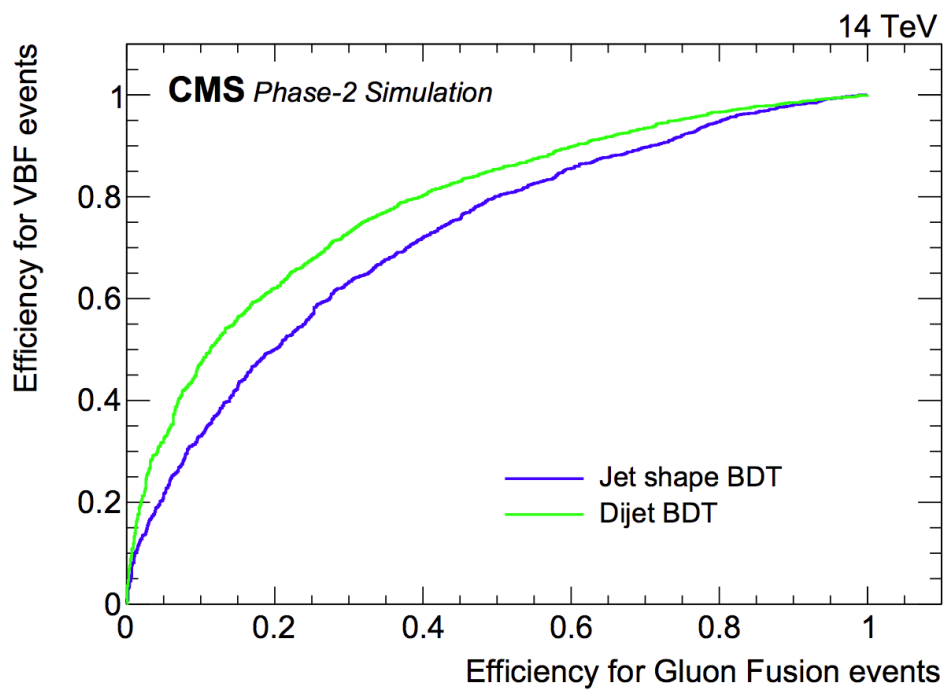

Figure 4: The selection efficiency for VBF and ggH events for two different BDT setups. Taken from [2].

One of the main benefits of the HGCAL upgrade will be the ability to separate real VBF jets from those originating from gluon emission. This is illustrated in a study of the two production modes in the $H \rightarrow \gamma \gamma$ decay channel. Two Boosted Decision Tree (BDT) classifiers are constructed, one using only jet shape variables and another including further jet and photon kinematic variables. The latter is very similar to one used in the existing Run 2 analysis. The performance of the jet shape only BDT, measured using the area under the ROC curve shown in Figure 4, is 0.71. This improves to 0.79 with the full kinematic information included, and is even higher than the Run 2 performance of 0.75 . With $3000 \mathrm{fb}^{-1}$ of data, a suitably chosen cut on this BDT would yield 750 signal events, with $75 \% \mathrm{VBF}$ purity, for a background of 678 events per $\mathrm{GeV}$, comparable to the Run 2 values.

Further results include the VBF $H \rightarrow \tau \tau$ analysis, where the visible di-tau mass is shown to be both robust against pileup and have similar resolution to the Run 2 detector. This resolution is crucial in being able to reduce the contamination of the irreducible $Z \rightarrow \tau \tau$ background, and therefore for maximising the sensitivity of the analysis.

\section{Beam tests}

To validate the design of the HGCAL and ensure its behaviour is well-modelled by simulation, beamtests have been conducted at both CERN and Fermilab sites in 2016 and 2017. Prototype silicon modules representative of those in both the CE-E and CE-H were built, with plastic scintillator tiles modified from an existing detector developed by the CALICE collaboration. Comparisons of the measured electron energy resolution and hadronic shower shape to simulation are shown in Figure 5. These prototypes were not the full design thickness, so the performance is not representative of the final detector. However the results constitute the first demonstration that the HGCAL 

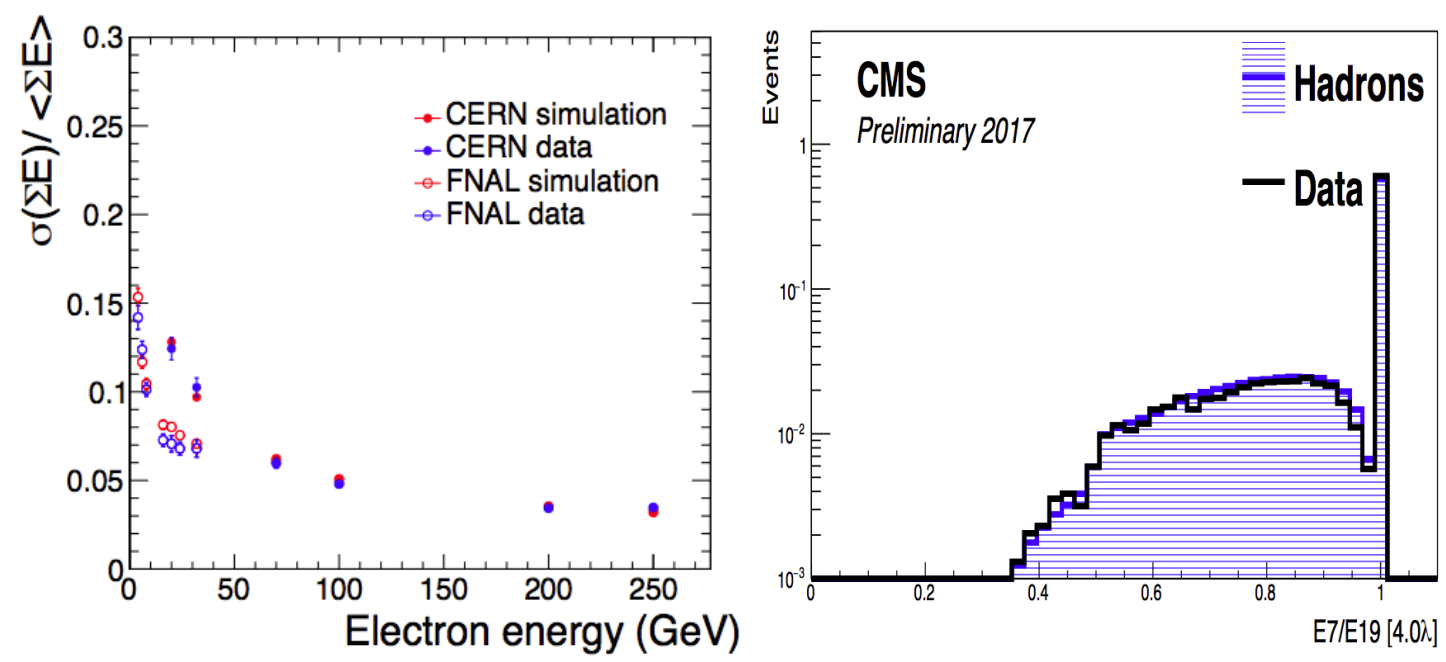

Figure 5: Left: electron energy distribution for both data and Monte Carlo simulation. Right: Transverse shower shape distribution in the CE-H.

behaves as predicted by simulation; observed distributions match those predicted by simulation to within 5\%. Furthermore, the tests confirm the intrinsic timing capabilities of the silicon sensors, with timing resolution measured to be less than 30ps. The timing performance of the silicon was also confirmed to be a function of $\mathrm{S} / \mathrm{N}$ only, and not degrade with increasing radiation exposure.

\section{Conclusion}

The HGCAL project is designed to replace the existing CMS endcap calorimeters and withstand the harsh HL-LHC conditions for its full lifetime. Offering unprecedented granularity in lateral and longitudinal directions, it is expected to provide detailed three-dimensional images of showers in addition to precision timing information from the silicon sensors. This will allow for powerful pileup rejection, sophisticated pattern recognition within CMS particle flow, and precise energy measurements. The recent technical design report showcased the potential of the detector, and between now and installation in 2026 much work remains to be done to overcome the many technical challenges ahead.

\section{References}

[1] CMS collaboration, S. Chatrchyan et al., The CMS Experiment at the CERN LHC, JINST 3 (2008) S08004.

[2] CMS Collaboration, The Phase-2 Upgrade of the CMS Endcap Calorimeter, Tech. Rep. CERN-LHCC-2017-023. CMS-TDR-019, CERN, Geneva, Nov, 2017.

[3] CMS collaboration, A. M. Sirunyan et al., Particle-flow reconstruction and global event description with the CMS detector, JINST 12 (2017) P10003 [1706.04 965].

[4] A. Rodriguez and A. Laio, Clustering by fast search and find of density peaks, Science 344 (2014) 1492 [http://science.sciencemag.org/content/344/6191/1492.full.pdf]. 